\title{
BreastCare
}

\section{Pathway Oriented Therapy in Breast Cancer - a Light at the End of the Tunnel!}

\author{
Eugen Ruckhäberle Tanja Fehm \\ Department of Obstetrics and Gynecology, Heinrich-Heine-University Düsseldorf, Düsseldorf, Germany
}

\begin{abstract}
Breast cancer (BC) is the most common female cancer and the second leading cause of cancer death in women [1]. BC is an extraordinarily heterogeneous disease, composed of distinct intrinsic subtypes, which may be defined by gene expression profiling or immunohistochemistry biomarkers [2]. After the groundbreaking research data of Perou et al. [3], we now classify breast cancer into 4 intrinsic subtypes - luminal A and B, HER2 enriched and basal-like. In the last years, extensive research has been done on triple-negative breast cancer. Using gene expression profiling, the triple-negative subgroup was re-classified into 6 subtypes: basal-like 1 and 2, im-
\end{abstract} munomodulatory, mesenchymal, mesenchymal stem-like, and luminal androgen receptor subtype [4]. Those subtypes describe well defined cancer subgroups that differ in molecular, pathological, and prognostic characteristics. In 2000, and updated in 2011, Hanahan and Weinberg [5] postulated 6 hallmarks of cancer and at least 2 emerging hallmarks that are necessary for tumor growth and / or invasion. All those hallmarks include distinct intracellular signaling pathways that are enhanced or blocked during tumor progression. Some of those signaling cascades are well investigated, others are not yet completely understood. Besides a specific signaling pathway from surface receptors to the nucleus in certain tumor subtypes, there are some crosslinks between various pathways, e.g. ER and EGF receptor pathways. Crosslinking can result in enhancement or attenuation of the cell signal leading to therapy resistance and tumor growth. After years of treatment options solely based on endocrine and cytotoxic treatments, targeted and pathway-oriented therapies found their way into clinical practice. This became only possible by an increasing understanding of the molecular details of signaling pathways linked to initiation, invasion, and migration. Understanding the complex mechanisms of intracellular and intranuclear signaling is the key in the development of pathway oriented therapies.

Nonetheless, a persisting major medical need is the detection of predictive markers for those pathway oriented therapies. So far, only the estrogen and HER2 receptors are established and implemented in clinical routine for the identification of patients who will benefit from certain therapies. Therefore, translational research still needs to concentrate on the detection and evaluation of new, robust, and reliable predictive markers for those costly therapies.

This issue of BREAST CARE focusses on pathway oriented therapy and aims to elucidate 3 major pathways:

While checkpoint inhibition is established and approved in the treatment of metastatic melanoma, renal cell cancer, and NSCLC, the impact of immune checkpoint inhibition in breast cancer is still under evaluation in different clinical trials. Hartkopf and coauthors [6] review the existing data on PD1 and PDL1 blockade and discuss the possible role of checkpoint inhibitors in treatment concepts for breast cancer.

One of the milestones in breast cancer diagnosis and therapy has been the detection of epidermal growth factor cell surface receptors (HER1-4) and HER2 targeted therapies including trastuzumab, pertuzumab, and T-DM1. Stickler et al. [7] summarize the recent treatment strategies in primary and metastatic HER2-positive breast cancer.

The estrogen, HER2 and insulin-like growth factor (IGF) receptor signaling pathways are linked to the PI3K/AKT/mTOR pathway which is the most frequently altered pathway in breast cancer. The PI3K/AKT/mTOR pathway mediates e.g. survival, cell cycle, and cell growth. Crosstalk between these different signaling cascades is crucial for the development of an endocrine or anti-HER2 resistance. Lux et al. [8] elucidate the data about the PI3 kinase pathway and provide a detailed summary of new drugs currently under clinical investigation.

Based on the understanding of the hallmarks in cancer, we have learned a lot about the impact of various pathways in breast cancer. Only if we understand the molecular details, we can develop new pathway oriented therapies and implement them in clinical routine to increase therapy sensitivity and overcome therapy resistance.

\section{Disclosure Statement}

The authors have nothing to disclose.

\section{KARGER}

(c) 2016 S. Karger GmbH, Freiburg

Fax +497614520714
Prof. Dr. med. Tanja Fehm 


\section{References}

1 Siegel RL, Miller KD, Jemal A: Cancer statistics, 2016. CA Cancer J Clin 2016;66:7-30.

2 Coates AS, Winer EP, Goldhirsch A, Gelber RD, Gnan M, Piccart-Gebhart M, Thürlimann B, Senn HJ; Panel Members: Tailoring therapies - improving the management of early breast cancer: St Gallen International Expert Consensus on the Primary Therapy of Early Breast Cancer 2015. Ann Oncol 2015;26:1533-1546.

$\checkmark 3$ Perou CM, Sørlie T, Eisen MB, van de Rijn M, Jeffrey SS, Rees CA, Pollack JR, Ross DT, Johnsen H, Akslen LA, Fluge O, Pergamenschikov A, Williams C, Zhu SX, Lønning PE, Børresen-Dale AL, Brown PO, Botstein D: Molecular portraits of human breast tumours. Nature 2000;406:747-752.
Lehmann BD1, Bauer JA, Chen X, Sanders ME, Chakravarthy AB, Shyr Y, Pietenpol JA: Identification of human triple-negative breast cancer subtypes and preclinical models for selection of targeted therapies. J Clin Invest 2011;121:2750-2767.

5 Hanahan D, Weinberg RA: Hallmarks of cancer: the next generation. Cell. 2011 Mar 4;144:646-674

6 Hartkopf AD, Taran FA, Wallwiener M, Walter CB, Krämer B, Grischke E-M, Brucker, S: PD-1 and PD-L1 immune checkpoint blockade to treat breast cancer. Breast Care 2016;11: DOI:10.1159/000453569.
Stickeler E, Iborra S: HER2 orientated therapy in early and metastatic breast cancer. Breast Care 2016;11: DOI:10.1159/000453603.

8 Lux MP, Fasching PA, Schrauder MG, Hein A, Jud SM, Rauh C, Beckmann MW: The PI3K pathway: backgrounds and treatment approaches. Breast Care 2016;11: DOI:10.1159/000453133. 